\title{
Assessment of Health of River Ganga at Varanasi, India
}

\author{
Sonali Saxena $†$ and Prabhat Kumar Singh
}

Department of Civil Engineering, Indian Institute of Technology (Banaras Hindu University), Varanasi, India

†Corresponding author: Sonali Saxena; sonali16d@gmail.com

Nat. Env. \& Poll. Tech.

Website: www.neptjournal.com

Received: 01-10-2019

Revised: 29-10-2019

Accepted: $26-11-2019$

Key Words:

River health index

River health condition

River health assessment

Biotic indicators

River Ganga

\begin{abstract}
World across, there is an increasing concern about river health. In India, Ganga River Basin Management Plan (GRBMP) 2015 considered River Ganga as an ecological entity. This paper attempts to present a framework for river health assessment in India and discusses its applicability for River Ganga near Varanasi. In the proposed framework, the River Health Condition (RHC) is assessed through the calculation of River Health Index (RHI) on a 0-100 scale and categorized as Acceptable or Poor. RHI is calculated by using selected parameters/indices normalized on the $0-5$ scale based on their critical and target values. River Health is presented through a coloured circumscribed pentagon each of whose side represents one of five indicator groups: i. Organo-electrolytic-bacterial qualities. ii. Nutrients, iii. Algae, iv. Macroinvertebrates, and v. Fish. Application of the proposed framework has been tested and explained using observed data for four seasons per year for two years from five locations of River Ganga near Varanasi. The colour of circumscribing pentagon reflects overall river health condition at a given location and each side of pentagon reflects health score concerningto one indicator group. The analyses indicate that the health of River Ganga near Varanasi is improving with time. The river health is found at its best level during Spring season and unstable during Post Monsoon period at most of the locations. The severely reduced RHI indicate "Overstressed" condition of River Ganga at the confluence points of River Assi and Varuna, which are evidenced by the presence of pollution tolerant biotic species. There are clear stretches of the river near outfall points which are nutrients rich and organically polluted causing poor health of river showing a disturbed balance of biotic species. Indicator group score based RHI gives a clear identification of critical parameters which may be used in strategic planning for river health restoration.
\end{abstract}

\section{INTRODUCTION}

Since ages, rivers have been taken as a source of water. Seeing its importance, the Government of India declared River Ganga as the National River in the year 2008 (MoWR 2014). However, the river is getting polluted from many sources, including domestic sewage, industrial wastewaters and surface run off from fields. Different types of pollutants enter the river system which affects the water quality in turn affecting the aquatic organisms present in the riverine environment. For most of the times, water resource managers understand water quality by measuring a few physico-chemical and biological characteristics and suggest its suitability for various beneficial uses. Ganga River Basin Management Plan (GRBMP) 2015 prepared by Government of India has considered River Ganga as 'ecological entity'. From this perspective, researchers and experts in the field have been feeling the need to define river health and parameters for its assessment. It has been realized that in addition to physico-chemical and bacteriological water quality parameters, it is necessary to include aquatic organisms, such as algae, macroinvertebrates and fishes present in the riverine environment as indicators to define river health. These aquatic organisms convey the integrated and continuous characteristics of water quality. Therefore, many experts (Hawkes 1979, Sladecek 1979, Tittizer \& Koth 1979, Hellawell 1986, Rosenberg \& Resh, 1993, Allan 1995) are of the view that algae, macroinvertebrates and fish should be considered as suitable indicators for assessing the health of rivers. The Australia-China Environment Development Partnership (ACEDP) Report 2011, under the title 'Assessment of River Health in Liao River Basin, Taizi Sub-catchment', China is a good example in this line of thinking. In the study, from the whole spectrum of water quality characteristics, 15 parameters have been selected and divided into five categories: i. Physical and Chemical parameters (P\&C), ii. Nutrients (NT), iii. Algae (A), iv. Macroinvertebrates $(\mathrm{MI})$, and v. Fish $(\mathrm{F})$. Five $\mathrm{P} \& \mathrm{C}$ parameters included Electrical Conductivity (EC), Dissolved Oxygen (DO), Biochemical Oxygen Demand (BOD), Chemical Oxygen Demand (COD) and Phenols. Two NT parameters include Ammonia- $\mathrm{N}\left(\mathrm{NH}_{4}-\mathrm{N}\right)$ and Total Phosphorus (TP). Two algal indices include a multi-metric Index of Biotic Integrity (A_BI2) and Berger-Parker dominance index (A_BP). Three macroinvertebrate indices include family-level rich- 
ness (M_S), BMWP score (M_BMWP) and family-level EPT richness (M_EPT_S). Three fish indices include family-level richness (F_S), fish index of biotic integrity (F_BI) and Berger Parker index (F_BP). River health assessment has been made using these 15 parameters. River Health Report Card (2012) has been presented in pictorial form in the shape of a pentagon, each side of pentagon depicting river health in terms of one category of characteristics, i.e. P\&C, nutrients, algae, macroinvertebrates and fish. Under Indian conditions, it has been observed that organic pollution is predominant in rivers and almost all the surface water sources have the presence of Coliform group of bacteria to some extent (Bhardwaj 2005). Accordingly, Singh \& Saxena (2018) proposed calculation of River Health Index (RHI) based on suitably selected indicators and parameters. In this framework, Faecal Coliform (FC) was included (replacing Phenol taken in Australia-China Study) among five P\&C category characteristics, NT parameters were increased from 2 to 3 by including Total Nitrogen (TN) as this is more readily available data. Among biotic indicators, identification and counting based simple indices for algae, macroinvertebrate and fish which could be performed by non-experts also, were selected and used. This included Algal Palmer Genus Pollution Index (APPI), (index for algae), Macroinvertebrate Shannon Weiner Diversity Index (M_SW), Macroinvertebrate BMWP score (M-BMWP) (two indices-for macroinvertebrates), and Fish Family level Richness Index (F_S), Shannon Weiner Diversity index (FSW) (two indices for fishes).

The present work is an attempt to interpret and understand the above framework of river health assessment for Ganga at Varanasi taking water quality and biotic indicators together into consideration. The framework calculates River Health Index (RHI) based on five indicator group scores, using a total of 13 parameters/indices. Organo-Electrolytic-Bacterial (OEB) group has five parameters (BOD, COD, DO, Electrical Conductivity and Fecal Coliform values), Nutrients (NT) group consists of 3 monitoring characteristics: $\mathrm{NH}_{3}-\mathrm{N}$, Total-Nitrogen and Total Phosphorus measurements. In addition, algae, macroinvertebrates and fishes are represented using five indices suitable for their groups. River Health Condition (RHC) has been classified as Acceptable (Excellent, Very Good, Good) and Poor (Stressed, Overstressed, Critical and Sick/Dead) based on RHI.

\section{MATERIALS AND METHODS}

\section{Study Area}

The Varanasi district lies in Uttar Pradesh (UP) province of India between $82^{\circ} 56^{\prime} \mathrm{E}-83^{\circ} 03^{\prime} \mathrm{E}$ and $25^{\circ} 14^{\prime} \mathrm{N}-25^{\circ} 23.5^{\prime} \mathrm{N}$. The city of Varanasi is situated in the middle stretch of Ganges basin in the eastern part of Uttar Pradesh. The crescent-shape Ganges banks are all through the left side of the city (Fig. 1). The samples of the river water were collected for two years from September 2016 to May 2018 during four seasons: Post Monsoon (16 Sep-15 Nov), Winter (16 Nov-15 Jan), Spring (16 Jan-15 Mar) and Summer (16 Mar-15 May) from 5 locations along the Ghats, starting from Saamne Ghat (upstream of confluence point of river Assi with Ganga) to the confluence point of River Varuna with Ganga in the downstream side of the city. The samples were collected from Saamne Ghat (L1), the confluence of (C/O) Assi Nala with Ganga (L2), Dashashwamedh Ghat (L3), Raaj Ghat (L4) and C/O Varuna with Ganga (L5) stretched in a length of around $7 \mathrm{~km}$.

\section{The Framework of River Health Index (RHI) Calculation}

In this study, the framework of River Health Index (RHI) calculation is based on scores of aquatic environment parameters divided into five indicator groups: i. Organo-Electrolytic-Bacterial (OEB) group (comprising of EC, DO, BOD, COD and FC, Total 5 parameters), ii. Nutrients (NT) group (consisting of $\mathrm{NH}_{3}-\mathrm{N}$, TN and TP, Total= 3 parameters), iii. Algae (Genus level Algal Palmer Pollution Index (APPI) One index), iv. Macroinvertebrate (Shannon Weiner Diversity index (MSW) and Macroinvertebrate BMWP score (MBMWP), Two Indices), and v. Fishes (Family level Fish
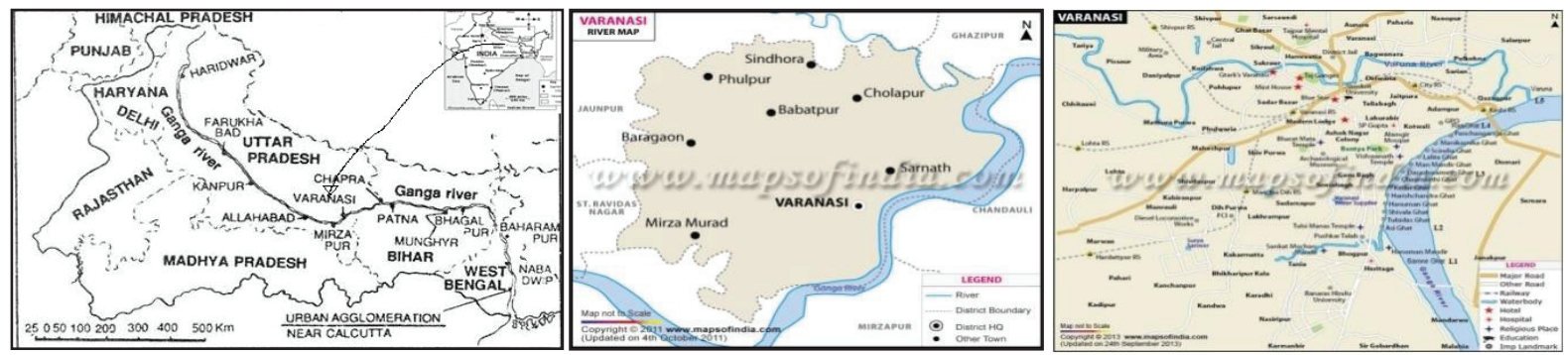

Fig.1: River Ganga near Varanasi and sampling locations. 
Species richness index (FS) and Shannon Weiner Diversity index (FSW), Two indices).

The group scores of Organo-Electrolytic-Bacterial (OEB), Nutrient (NT), Algae (A), Macroinvertebrate (MI) and Fish $(\mathrm{F})$ indicators were used to calculate the River Health Index (RHI) by the following formula:

River Health Index (RHI)

$$
\begin{aligned}
= & {\left[\left(\mathrm{OEB} \mathrm{x} \mathrm{w}_{1}\right)+\left(\mathrm{NT} \mathrm{x} \mathrm{w}_{2}\right)+\left(\mathrm{A} \mathrm{x} \mathrm{w}_{3}\right)+\left(\mathrm{MI} \mathrm{x} \mathrm{w} \mathrm{w}_{4}\right)\right.} \\
& \left.+\left(\mathrm{F} \mathrm{x} \mathrm{w}_{5}\right)\right]
\end{aligned}
$$

Where, $\mathrm{OEB}=$ Organo-Electrolytic-Bacterial indicator group score, $\mathrm{NT}=$ Nutrient indicator group score, $\mathrm{A}=$ Algal indicator group score, $\mathrm{MI}=$ Macroinvertebrate indicator group score, and $\mathrm{F}=\mathrm{Fish}$ indicator group score and $\mathrm{w}_{1}, \mathrm{w}_{2}$, $\mathrm{w}_{3}, \mathrm{w}_{4}$ and $\mathrm{w}_{5}$ are weightages given to different groups (Table 1). The OEB and NT group indicators are normally affected by short term fluctuations, whereas biotic indicators such as algae, macroinvertebrates and fish are long term integrators of river health. Therefore, the biotic indicators should contribute more heavily towards an overall RHI. With similar reasoning, macroinvertebrates and fish indicators are weighted more heavily than algal indicators as they are longer lived than algae (Leigh et al. 2012).

River Health Condition (RHC) is defined based on indicator group score andRiver Health Index (RHI) and colour code is given for visual representation, as shown in Table 2.
The river health is considered Acceptable or Poor if RHI is greater than or less than 60. Acceptable river health may be of three categories: Good (RHI 60-70), Very Good (RHI 70-80) or Excellent (RHI>80). With poor river health, rivers may be categorized as Stressed (RHI 50-60), Overstressed (RHI 40-50), Critical (RHI 20-40) or Sick/Dead (RHI $\leq 20)$. The river health is pictorially represented by a circumscribed pentagon in which colour of each side represents one indicator group score of water quality in the river environment and the colour of the circumscribing pentagon represents the river health condition (RHC) based on overall River Health Index (RHI) for the site (Fig. 2).

\section{Sample Collection and Analyses}

Samples for organo-electrolytic-bacterial, nutrient, algae and macroinvertebrate analyses were collected from 5 locations along the Ghats of Varanasi during post-monsoon (16Sep$15 \mathrm{Nov})$, winter (16Nov-15Jan), spring (16Jan-15Mar) and summer (16Mar-15May) for two years 2016-2017 and 20172018. Three grab samples were collected during morning hours between 8.00 a.m. and 11.00 a.m. from each location and mixed to form a compound sample for that location once every month and grouped in seasons. The total number of samples for which analyses have been performed is 360 (120 each for Organo-Electrolytic-Bacterial, Nutrient, Algae and Macroinvertebrate). Some parameters such as electrical conductivity (EC), dissolved oxygen (DO) (includ-

\begin{tabular}{|c|c|c|c|c|c|}
\hline & Indicator Group & Parameters/Indices & No. of Parameters/Indices & Weight factor & $\begin{array}{l}\text { Weight factor } \\
\text { given in the present study }\end{array}$ \\
\hline 1 & Organo-Electrolytic-Bacterial (OEB) & $\mathrm{EC}, \mathrm{DO}, \mathrm{BOD}, \mathrm{COD}, \mathrm{FC}$ & 5 & $\mathrm{w}_{1}$ & 0.15 \\
\hline 2 & Nutrient Score (NT) & $\mathrm{NH}_{3}-\mathrm{N}, \mathrm{TN}, \mathrm{TP}$ & 3 & $\mathrm{w}_{2}$ & 0.15 \\
\hline 3 & Algae Score (A) & APPI & 1 & $\mathrm{w}_{3}$ & 0.20 \\
\hline 4 & Macroinvertebrate Score (MI) & MSW, MBMWP & 2 & $\mathrm{w}_{4}$ & 0.25 \\
\hline \multirow[t]{2}{*}{5} & Fish Score $(\mathrm{F})$ & FS, FSW & 2 & $\mathrm{w}_{5}$ & 0.25 \\
\hline & Total & & 13 & & 1.00 \\
\hline
\end{tabular}

Table 1: Weightage of different indicator groups.

Table 2: River Health Condition (RHC) based on Indicator Group Score and River Health Index (RHI).

\begin{tabular}{|llll|}
\hline River Health & Indicator Group Score/ RHI Score & RHC & Colour Code \\
\hline \multirow{3}{*}{ Acceptable } & $>80$ & Excellent & Blue \\
& $70-80$ & Very Good & Green \\
\hline & $60-70$ & Good & Yellow \\
Poor & $50-60$ & Stressed & Grey \\
& $40-50$ & Over Stressed & Red \\
& $20-40$ & Critical & Black \\
\hline
\end{tabular}




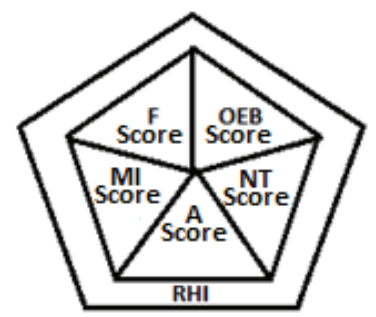

Fig. 2: Circumscribed pentagon for the river health condition and river health index.

ing temperature, and $\mathrm{pH}$ ) were recorded on the site using a multi-parameter analyser. Others including biochemical oxygen demand (BOD) by 5-day BOD test, chemical oxygen demand (COD) by closed reflux titrimetric method (Hanna Instrument), faecal coliforms (FC) by multiple tube fermentation technique and nutrient parameters, such as ammonia-nitrogen $\left(\mathrm{NH}_{3}-\mathrm{N}\right)$ by titrimetric method, total nitrogen (TN) and total phosphorous (TP) by stannous chloride method were tested in the laboratory as per the standard methods (APHA 2005).

The samples for algal analyses were collected in bottles and preserved in $4 \%$ formalin solution and transported to the laboratory for identification using the microscope.

The Genus based Algal Palmer Pollution Index (APPI) was calculated using Table 3 (Palmer 1969, Nandan \& Patel 1986). Pollution classification of Palmer (1969) suggests that APPI $=0-10$ represents lack of organic pollution, 10-15 indicates moderate pollution, 15-20 marks probable high organic pollution, and 20 or more confirms high organic pollution.

The macroinvertebrate samples were collected using standard D-frame dip net having 500-micron opening. Ben- thic macroinvertebrates were collected systematically from all available instream habitats by kicking the substrate and jabbing with a D-frame dip net. The samples were preserved in $4 \%$ formalin solution and transported to the laboratory for further examination. In the laboratory, the samples were rinsed thoroughly with pure water to remove the preservative. Collected samples were examined and counted using the hand lens and microscope. The macroinvertebrates were identified to the lowest possible taxonomic level using standard taxonomic literature APHA (2005), Gerber \& Gabriel (2002), Barbour et al. (1999), Merritt \& Cummins (1996), William \& Feltmate (1992), Pennak (1989), Durrand \& Leveque (1981), Tonapi (1980), Pennak (1978) and Needham \& Needham (1969). The Macroinvertebrate Shannon Weiner Diversity Index (MSW) was calculated as follow:

$$
\mathrm{MSW}=-\Sigma \text { pi.ln } \mathrm{pi}
$$

Where, $\mathrm{pi}=\mathrm{S} / \mathrm{N}, \mathrm{S}=$ Number of individual of particular specie, $\mathrm{N}=$ Total number of individuals of all species in the sample.

Macroinvertebrate Biological Monitoring Working Party (MBMWP) score was calculated based on the presence of

Table 3: Genus based Algal Palmer Pollution Index (APPI) (Palmer 1969, Nandan \& Patel 1986).

\begin{tabular}{|llll|}
\hline Genus & Pollution Index & Genus & Pollution Index \\
\hline Anacystis & 1 & Micractinium & 1 \\
Ankistrodesmus & 2 & Navicula & 3 \\
Chlamydomonas & 4 & Nitzschia & 3 \\
Chlorella & 3 & Oscillatoria & 5 \\
Closterium & 1 & Pandorina & 1 \\
Cyclotella & 1 & Phacus & 2 \\
Euglena & 5 & Phormidium & 1 \\
Gomphonema & 1 & Scenedesmus & 4 \\
Lepocinclis & 1 & Stigeoclonium & 2 \\
Melosira & 1 & Synedra & 2 \\
Anabaena & 1 & & \\
\hline
\end{tabular}

Note: Pollution classification of Palmer (1969) suggest that APPI = 0-10 represents lack of organic pollution, 10-15 indicates moderate pollution, 15-20 marks probable high organic pollution, and 20 or more confirms high organic pollution. 
taxonomical class and families using Table 4 (De Zwart \&Trivedi 1994).

The two fish indices, i.e. Fish Species Index (FS) and Shannon Weiner Diversity Index (FSW) were calculated based on species count and process suggested in the available literature (Das et al. 2013, Dwivedi et al. 2016).

The 'Target Value' and 'Critical Value' for the indicators were obtained from available literature as given in Table 5 .

The values of individual parameter/indices obtained from sample analysis are used to calculate the score of the parameter/index at a particular location. For making the score calculation more precise, the full range between 'Target value' and 'Critical value' has been divided into five zones on a 0-5 scale, as given in Table 6. According to this, if at a particular site, the observed value of an indicator is better than or equal to the target value, the site would have an indicator score 5 and if the observed value is less than the critical value, the indicator score would be 0 .

The group indicator score is calculated by aggregating the parameters/indices score of each group.

\section{Indicator Group Score}

$=[\Sigma$ scores of parameters or indices $/(5 \times$ No. of parameters or indices in the group) $] \times 100$

Table 4: Biological Monitoring Working Party (BMWP) Score (De Zwart \& Trivedi 1994).

\begin{tabular}{|c|c|c|}
\hline Taxonomical Class & Taxonomical Families & BMWP Score \\
\hline Ephemeroptera & Heptogeniidae, Leptophlebiidae, Ephemerellidae, Ephemeridae, Potoaminthidae, Siphonuridae & 10 \\
\hline Plecoptera & Leuctridae, Capniidae, Perlodidae, Perlidae, Taeniopterygidae & \\
\hline Hemiptera & Aphelocheiridae & \\
\hline Trichoptera & Leptoceridae, Goeridae, Lepidostomatidae, Brachycentridae, Sericostomatidae & \\
\hline Odonata & Lestidae, Gomphidae, Cordulegasteridae, Aeschnidae, Corduliidae, Libellulidae, Plathycnemididae & 8 \\
\hline Trichoptera & Psychomyiidae, Philopotomidae & \\
\hline Ephemeroptera & Caenidae & 7 \\
\hline Plecoptera & Nemouridae & \\
\hline Trichoptera & Rhyacophilidae, Polycentropodidae, Limnephilidae & \\
\hline Mollusca & Ancylidae, Hydrobiidae, Neritidae, Viviparidae, Thiaridae, Bithynidae, Unionidae & 6 \\
\hline Trichoptera & Hydroptilidae & \\
\hline Crustacea & Palaemonidae, Atydae, Gammaridae & \\
\hline Polychaeta & Nereidae, Nephthyidae & \\
\hline Odonata & Coenagriidae, Agriidae & \\
\hline Hemiptera & $\begin{array}{l}\text { Mesovelidae, Hydrometridae, Gerridae, Nepidae, Naucoridae, Notonectidae, Pleidae, Corixidae, Veliidae, } \\
\text { Hebridae, Belestomatidae }\end{array}$ & 5 \\
\hline Coleoptera & $\begin{array}{l}\text { Haliplidae, Hygrobidae, Dytiscidae, Gyrinidae, Hydrophilidae, Noteridae, Helodidae, Dryopidae, El- } \\
\text { minthidae, Psephenidae }\end{array}$ & \\
\hline Trichoptera & Hydropsychidae & \\
\hline Diptera & Tipulidae, Culicidae, Blepharoceridae, Simulidae & \\
\hline Planaria & Planariidae, Dendroceolidae & \\
\hline Ephemeroptera & Baetidae & 4 \\
\hline Megaloptera & Sialidae & \\
\hline Hirudinea & Piscicodidae & \\
\hline Mollusca & Lymnaeidae, Planorbidae, Sphaeridae & 3 \\
\hline Hirudinea & Glossiphonidae, Hirudidae, Erpobdellidae & \\
\hline Planaria & Dugesiidae & \\
\hline Crustacea & Asselidae & \\
\hline Diptera & Chironomidae, Syrphidae & 2 \\
\hline Oligochaeta & All families & 1 \\
\hline
\end{tabular}


Table 5: Target and Critical values for parameters/indices in a river environment.

\begin{tabular}{|c|c|c|c|c|c|}
\hline S.N. & Indicator Group & Parameter/ Indices & Target value & Critical value & Source \\
\hline \multirow[t]{5}{*}{1} & \multirow{5}{*}{$\begin{array}{l}\text { Organo-Electrolytic-Bac- } \\
\text { terial (OEB) }\end{array}$} & i. EC $(\mu \mathrm{mhos} / \mathrm{cm})$ & $\leq 400$ & $>1500$ & EHMP (2010); (Anon 2000) \\
\hline & & ii. $\mathrm{DO}(\mathrm{mg} / \mathrm{L})$ & $\geq 7$ & $<3$ & UNECE (1994) \\
\hline & & iii. BOD (mg/L) & $\leq 3$ & $>8$ & CPCB (2015)(Existing); CPCB (2002) \\
\hline & & iv. $\mathrm{COD}(\mathrm{mg} / \mathrm{L})$ & $\leq 30$ & $>80$ & Assumed (Currently no limit is available). \\
\hline & & v. FC (MPN/100mL) & $\leq 500$ & $>2500$ & CPCB (2015) (Existing) \\
\hline \multirow[t]{3}{*}{2} & \multirow[t]{3}{*}{ Nutrients (NT) } & i. $\mathrm{NH}_{3}-\mathrm{N}(\mathrm{mg} / \mathrm{L})$ & $\leq 0.3$ & $>1.5$ & CPCB (2002); MEP (2008) \\
\hline & & ii. $\mathrm{TN}(\mathrm{mg} / \mathrm{L})$ & $\leq 0.5$ & $>2$ & Anon (2000); MEP (2008) \\
\hline & & iii. TP (mg/L) & $\leq 0.1$ & $>0.3$ & CPCB (2002) \\
\hline 3 & Algae (A) & i. Genus APPI & $\leq 10$ & $>20$ & Palmer (1969) \\
\hline \multirow[t]{2}{*}{4} & \multirow[t]{2}{*}{ Macroinvertebrate (MI) } & i. MSW & $>3.5$ & 0 & Kerkhoff (2010) \\
\hline & & ii. MBMWP (Saprobic) & $>7$ & 0 & СРCB (2015) \\
\hline \multirow[t]{2}{*}{5} & \multirow[t]{2}{*}{ Fish $(F)$} & $\begin{array}{l}\text { i. Family Level Fish Species } \\
\text { Richness Index (FS) }\end{array}$ & $\geq 75$ & 0 & Das et al. (2013) \\
\hline & & ii. FSW & $>3.5$ & 0 & Das et al. (2013) \\
\hline
\end{tabular}

Table 6: Score of parameters/indices on a 0-5 scale.

\begin{tabular}{|c|c|c|c|c|c|c|c|c|}
\hline & Indicator Group & Parameter/Indices & Score & & & & & \\
\hline \multirow{4}{*}{1} & \multirow{4}{*}{$\begin{array}{l}\text { Organo-Elec- } \\
\text { trolytic-Bacte- } \\
\text { rial (OEB) }\end{array}$} & & 0 & 1 & 2 & 3 & 4 & 5 \\
\hline & & i. $\mathrm{EC}(\mu \mathrm{mhos} / \mathrm{cm})$ & $>1500$ & $1250-1500$ & $1000-1250$ & $750-1000$ & $400-750$ & $\leq 400$ \\
\hline & & ii. $\mathrm{DO}(\mathrm{mg} / \mathrm{L})$ & $<3$ & $3-4$ & $4-5$ & $5-6$ & $6-7$ & $\geq 7$ \\
\hline & & iii. BOD (mg/L) & $>8$ & $6.5-8$ & $5.0-6.5$ & $4.0-5.0$ & $3.0-4.0$ & $\leq 3$ \\
\hline \multirow{5}{*}{2} & \multirow{5}{*}{ Nutrients (NT) } & iv. $\mathrm{COD}(\mathrm{mg} / \mathrm{L})$ & $>80$ & $65-80$ & $50-65$ & $40-50$ & $30-40$ & $\leq 30$ \\
\hline & & v. FC (MPN/100 mL) & $>2500$ & $2000-2500$ & $1500-2000$ & $1000-1500$ & $500-1000$ & $\leq 500$ \\
\hline & & i. $\mathrm{NH}_{3}-\mathrm{N}(\mathrm{mg} / \mathrm{L})$ & $>1.5$ & $1.2-1.5$ & $0.9-1.2$ & $0.6-0.9$ & $0.3-0.6$ & $\leq 0.3$ \\
\hline & & ii. $\mathrm{TN}(\mathrm{mg} / \mathrm{L})$ & $>2$ & $1.6-2.0$ & $1.2-1.6$ & $0.8-1.2$ & $0.5-0.8$ & $\leq 0.5$ \\
\hline & & iii. TP (mg/L) & $>0.3$ & $0.25-0.3$ & $0.2-0.25$ & $0.15-0.2$ & $0.1-0.15$ & $\leq 0.1$ \\
\hline 3 & Algae (A) & i. APPI (Genus) & $>20$ & $18-20$ & $15-17$ & $13-14$ & $11-12$ & $\leq 10$ \\
\hline \multirow[t]{2}{*}{4} & \multirow{2}{*}{$\begin{array}{l}\text { Macroinverte- } \\
\text { brate (MI) }\end{array}$} & i. MSW & 0 & $0-1.0$ & $1.0-2.0$ & $2.0-3.0$ & $3.0-3.5$ & $>3.5$ \\
\hline & & ii. MBMWP (Saprobic) & 0 & $0-2.0$ & $2.0-4.0$ & $4.0-5.5$ & $5.5-7.0$ & $>7$ \\
\hline \multirow[t]{2}{*}{5} & Fish $(F)$ & i. FS (Species) & 0 & $1-15$ & $15-35$ & $35-55$ & $55-75$ & $\geq 75$ \\
\hline & & ii. FSW & 0 & $0-0.75$ & $0.75-1.5$ & $1.5-2.5$ & $2.5-3.5$ & $>3.5$ \\
\hline
\end{tabular}

\section{RESULTS AND DISCUSSION}

The list of algae and different macroinvertebrate families found at all five locations of sampling in Varanasi during the study period is listed in Tables 7 and 8 .

The range of values observed for different parameters/ indices at all 5 locations of Varanasi during four seasons of two years period is given in Table 9 .

The observed value of parameter/index is assigned a score between $0-5$ using Table 6 . The value of the indicator group score is calculated using Eqn. (2). Based on the indicator group score, the River Health Index (RHI) is calculated from Eqn. (1)and the River Health Condition (RHC) is categorized. The parameter/index score of the individual parameter/index, indicator group score, RHI and RHC at a particular location for two years (Sep 2016-May 2017 and Sep 2017- May 2018) is given in Table 10 .

From Table 10, it is observed that, based on RHI calculated through the proposed framework, health condition of River Ganga at Varanasi is acceptable and under 'Good' category 
Table 7: Algal groups and genera found at different locations of River Ganga near Varanasi (Sept 2016-May 2018).

\begin{tabular}{|c|c|c|c|}
\hline Group & Genus & Group & Genus \\
\hline \multirow{15}{*}{ Bacillariophyceae } & Achnanthes & \multirow{12}{*}{ Chlorophyceae } & Mougeotia \\
\hline & Asterionella & & Oedogonium \\
\hline & Cocconeis & & Oocystis \\
\hline & Cyclotella & & Palmella \\
\hline & Cymbella & & Pediastrum \\
\hline & Fragilaria & & Scenedesmus \\
\hline & Gomphonema & & Sphaerocystis \\
\hline & Melosira & & Spirogyra \\
\hline & Navicula & & Staurastrum \\
\hline & Nitzschia & & Stigeoclonium \\
\hline & Stauroneis & & Tetraspora \\
\hline & Surirella & & Tribonema \\
\hline & Synedra & \multirow{13}{*}{ Cyanophyceae } & Ulothrix \\
\hline & Tabellaria & & Volvox \\
\hline & & & Zygnema \\
\hline \multirow{11}{*}{ Chlorophyceae } & Actinastrum & & \\
\hline & Ankistrodesmus & & Anabaena \\
\hline & Chlorella & & Anacystis \\
\hline & Chlorococcum & & Cylindrospermum \\
\hline & Cladophora & & Lyngbya \\
\hline & Clostridium & & Merismopedia \\
\hline & Coelastrum & & Oscillatoria \\
\hline & Crucigenia & & Phormidium \\
\hline & Draparnaldia & & Spirulina \\
\hline & Hydrodictyon & & \\
\hline & Microspora & Xanthophyceae & Vaucheria \\
\hline
\end{tabular}

Table 8: Macroinvertebrate Groups and Families found at different locations in River Ganga near Varanasi (Sept 2016-May 2018).

\begin{tabular}{|c|c|c|c|}
\hline \multicolumn{2}{|r|}{ Taxonomical Group } & Taxonomical Families & Common names \\
\hline 1 & Coleoptera & $\begin{array}{l}\text { Dytiscidae } \\
\text { Elmidae } \\
\text { Hydrophilidae }\end{array}$ & $\begin{array}{l}\text { Diving Beetles } \\
\text { Riffle Beetles } \\
\text { Water scavenger Beetles }\end{array}$ \\
\hline 2 & Diptera & $\begin{array}{l}\text { Chironomidae } \\
\text { Culicidae } \\
\text { Psychodidae } \\
\text { Simuliidae } \\
\text { Syrphidae } \\
\text { Tabanidae } \\
\text { Tipulidae } \\
\text { Muscidae } \\
\end{array}$ & $\begin{array}{l}\text { Non biting Midges } \\
\text { Mosquito larvae } \\
\text { Moth fly } \\
\text { Black fly } \\
\text { Rat-tailed maggot } \\
\text { Horse fly } \\
\text { Crane fly } \\
\text { Muscid fly }\end{array}$ \\
\hline 3 & Ephemeroptera & Baetidae & May fly \\
\hline 4 & Mollusca & $\begin{array}{l}\text { Lymnaeidae } \\
\text { Physidae } \\
\text { Pilidae } \\
\text { Viviparidae }\end{array}$ & $\begin{array}{l}\text { Pond Snails } \\
\text { Pouch Snails } \\
\text { Apple Snail } \\
\text { Snails }\end{array}$ \\
\hline 5 & Oligochaeta & $\begin{array}{l}\text { Tubifex } \\
\text { Tubificidae }\end{array}$ & $\begin{array}{l}\text { Worms } \\
\text { Worms }\end{array}$ \\
\hline
\end{tabular}


Table 9: Range of values of parameters/indices observed in River Ganga near Varanasi in four seasons during Sept 2016-May 2018.

\begin{tabular}{|c|c|c|c|c|c|c|c|c|c|}
\hline \multirow[b]{2}{*}{$\begin{array}{l}\text { Parameter/ } \\
\text { Indices }\end{array}$} & \multirow{2}{*}{ Unit } & \multicolumn{4}{|c|}{$2016-2017$} & \multicolumn{4}{|c|}{$2017-2018$} \\
\hline & & $\begin{array}{l}\text { Post } \\
\text { Monsoon } \\
\text { (16Sep- } \\
15 \text { Nov) }\end{array}$ & $\begin{array}{l}\text { Winter } \\
\text { (16Nov- } \\
\text { 15Jan) }\end{array}$ & $\begin{array}{l}\text { Spring } \\
\text { (16Jan- } \\
\text { 15Mar) }\end{array}$ & $\begin{array}{l}\text { Summer } \\
\text { (16Mar- } \\
\text { 15May) }\end{array}$ & $\begin{array}{l}\text { Post } \\
\text { Monsoon } \\
\text { (16Sep- } \\
\text { 15Nov) }\end{array}$ & $\begin{array}{l}\text { Winter } \\
\text { (16Nov- } \\
\text { 15Jan) }\end{array}$ & $\begin{array}{l}\text { Spring } \\
\text { (16Jan- } \\
\text { 15Mar) }\end{array}$ & $\begin{array}{l}\text { Summer } \\
\text { (16Mar- } \\
\text { 15May) }\end{array}$ \\
\hline Temperature & ${ }^{\circ} \mathrm{C}$ & $23.0-24.5$ & $17.0-18.0$ & $22.1-23.5$ & $27.2-28.2$ & $23.4-24.9$ & $17.1-18.0$ & $22.3-23.8$ & $27.3-28.2$ \\
\hline $\mathrm{pH}$ & & $7.8-8.4$ & $8.2-8.8$ & $8.0-8.6$ & $7.5-7.9$ & $7.8-8.3$ & $8.1-8.6$ & 7.9-8.6 & $7.5-8.0$ \\
\hline EC & $\mu \mathrm{mhos} / \mathrm{cm}$ & $600-700$ & $265-300$ & $300-400$ & $420-900$ & $572-650$ & $293-372$ & $332-390$ & $420-1100$ \\
\hline DO & $\mathrm{mg} / \mathrm{L}$ & $3.9-6.5$ & $4.0-6.3$ & $4.1-6.3$ & $2.9-5.8$ & $4.2-6.7$ & $4.6-6.5$ & $4.2-6.3$ & $2.9-5.9$ \\
\hline BOD & $\mathrm{mg} / \mathrm{L}$ & $4.3-7.0$ & $4.2-6.2$ & $4.0-6.0$ & $4.3-6.8$ & $4.7-6.8$ & $3.9-6.0$ & $4.0-6.4$ & $4.4-6.9$ \\
\hline COD & $\mathrm{mg} / \mathrm{L}$ & $34-72$ & $38-68$ & $55-82$ & $60-100$ & $40-68$ & $42-78$ & $52-80$ & $52-110$ \\
\hline $\mathrm{FC}$ & MPN/100 mL & $1100-4200$ & $1500-3200$ & $1300-2200$ & $1800-3600$ & $1200-3100$ & $1200-2200$ & $1200-1900$ & $1600-3300$ \\
\hline $\mathrm{NH}_{3}-\mathrm{N}$ & $\mathrm{mg} / \mathrm{L}$ & $0.40-1.48$ & $0.50-1.50$ & $0.45-1.04$ & $0.90-2.01$ & $0.60-1.18$ & $0.38-0.60$ & $0.19-0.56$ & $0.90-1.50$ \\
\hline $\mathrm{TN}$ & $\mathrm{mg} / \mathrm{L}$ & $0.91-2.34$ & $1.35-2.50$ & $1.08-1.68$ & $1.37-2.40$ & $1.35-1.65$ & $0.68-1.38$ & $0.52-1.32$ & $1.35-2.00$ \\
\hline $\mathrm{TP}$ & $\mathrm{mg} / \mathrm{L}$ & $\begin{array}{l}0.160- \\
0.359\end{array}$ & $\begin{array}{l}0.195- \\
0.378\end{array}$ & $\begin{array}{l}0.145- \\
0.410\end{array}$ & $\begin{array}{l}0.210- \\
0.320\end{array}$ & $\begin{array}{l}0.202- \\
0.280\end{array}$ & $\begin{array}{l}0.195- \\
0.298\end{array}$ & $\begin{array}{l}0.125- \\
0.220\end{array}$ & $\begin{array}{l}0.160- \\
0.290\end{array}$ \\
\hline APPI(Genus) & & $13-16$ & $13-18$ & $13-17$ & $15-18$ & $13-16$ & $13-16$ & $13-15$ & $15-21$ \\
\hline MSW & & $0.70-1.09$ & $1.18-1.59$ & $1.33-1.58$ & $0.64-1.27$ & $0.99-1.20$ & $0.88-1.64$ & $1.41-1.65$ & $1.11-1.33$ \\
\hline MBMWP & & $3.00-4.50$ & $3.83-4.40$ & $3.83-4.80$ & $3.00-5.20$ & $4.25-4.33$ & $3.60-4.80$ & $4.20-5.00$ & $3.00-5.00$ \\
\hline FS & & 70 & 70 & 70 & 70 & 70 & 70 & 70 & 70 \\
\hline FSW & & 2.23 & 2.23 & 2.23 & 2.23 & 2.23 & 2.23 & 2.23 & 2.23 \\
\hline
\end{tabular}

Table 10: The Parameter/Index Score, Indicator Group Scores, RHI and RHC (Sep 2016-May 2018).

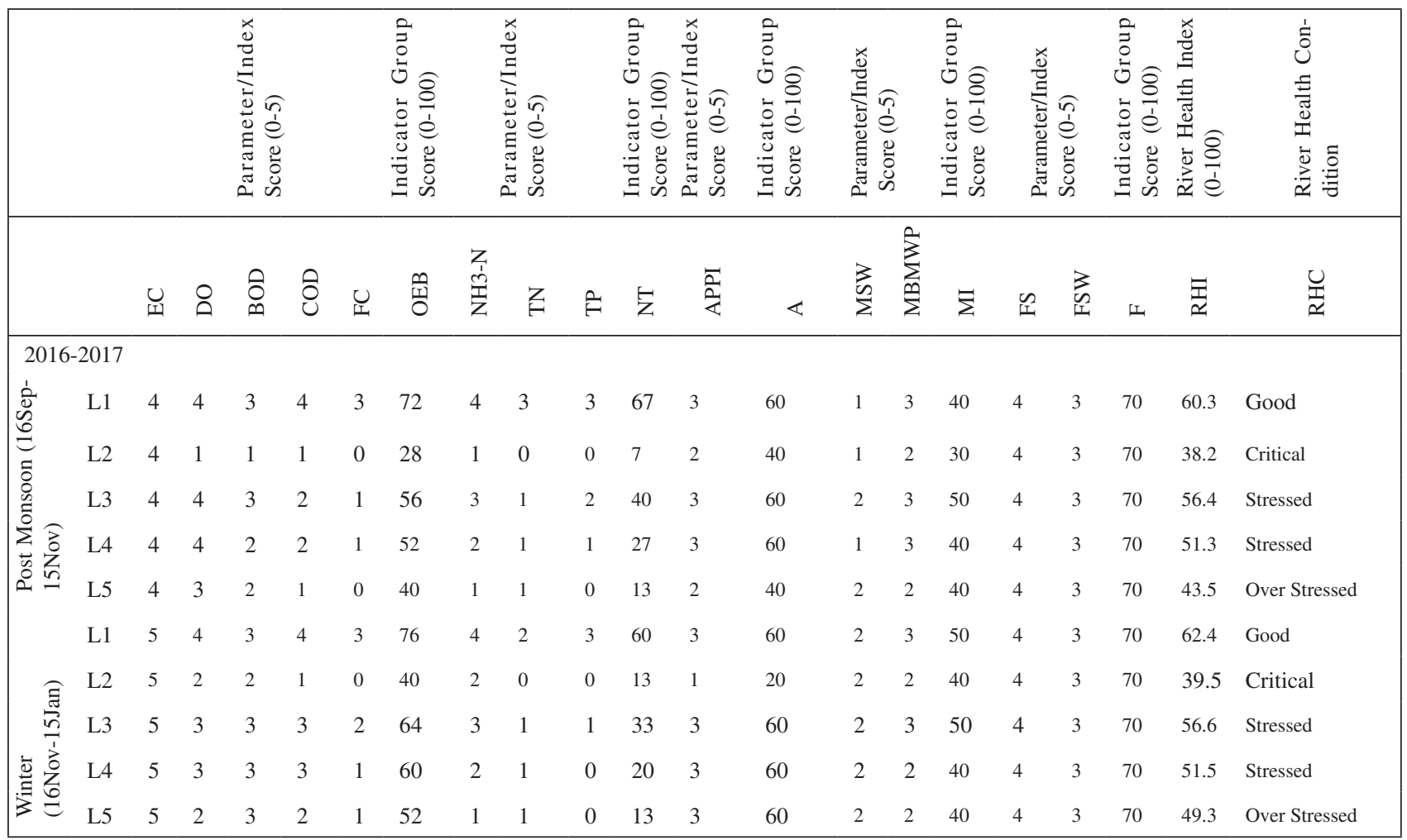

Table Cont.... 
Cont. Table...

\begin{tabular}{|c|c|c|c|c|c|c|c|c|c|c|c|c|c|c|c|c|c|c|c|c|c|}
\hline \multirow{5}{*}{ 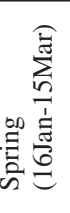 } & L1 & 5 & 4 & 4 & 2 & 3 & 72 & 4 & 3 & 4 & 73 & 3 & 60 & 2 & 3 & 50 & 4 & 3 & 70 & 63.8 & Good \\
\hline & $\mathrm{L} 2$ & 5 & 2 & 2 & 0 & 1 & 40 & 2 & 1 & 0 & 20 & 2 & 40 & 2 & 3 & 50 & 4 & 3 & 70 & 47.0 & Over Stressed \\
\hline & L3 & 5 & 4 & 3 & 2 & 2 & 64 & 4 & 3 & 1 & 53 & 3 & 60 & 2 & 3 & 50 & 4 & 3 & 70 & 59.6 & Stressed \\
\hline & L4 & 5 & 3 & 3 & 2 & 2 & 60 & 4 & 2 & 1 & 47 & 3 & 60 & 2 & 3 & 50 & 4 & 3 & 70 & 58.0 & Stressed \\
\hline & L5 & 5 & 3 & 2 & 1 & 1 & 48 & 4 & 2 & 0 & 40 & 3 & 60 & 2 & 2 & 40 & 4 & 3 & 70 & 52.7 & Stressed \\
\hline \multirow{5}{*}{ 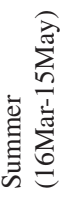 } & L1 & 4 & 3 & 3 & 2 & 2 & 56 & 3 & 2 & 2 & 47 & 2 & 40 & 2 & 3 & 50 & 4 & 3 & 70 & 53.4 & Stressed \\
\hline & L2 & 3 & 0 & 1 & 0 & 0 & 16 & 0 & 0 & 0 & 0 & 1 & 20 & 1 & 2 & 30 & 4 & 3 & 70 & 31.4 & Critical \\
\hline & L3 & 4 & 3 & 2 & 2 & 2 & 52 & 2 & 2 & 1 & 33 & 2 & 40 & 2 & 3 & 50 & 4 & 3 & 70 & 50.8 & Stressed \\
\hline & L4 & 3 & 2 & 2 & 0 & 1 & 32 & 2 & 1 & 2 & 33 & 2 & 40 & 1 & 3 & 40 & 4 & 3 & 70 & 45.3 & Over Stressed \\
\hline & L5 & 3 & 1 & 1 & 0 & 0 & 20 & 1 & 1 & 1 & 20 & 1 & 20 & 2 & 2 & 40 & 4 & 3 & 70 & 37.5 & Critical \\
\hline \multicolumn{22}{|c|}{ 2017-2018 } \\
\hline \multirow{5}{*}{ 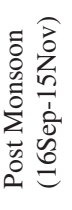 } & L1 & 4 & 4 & 3 & 4 & 3 & 72 & 4 & 2 & 2 & 53 & 3 & 60 & 2 & 3 & 50 & 4 & 3 & 70 & 60.8 & Good \\
\hline & L2 & 4 & 2 & 1 & 2 & 0 & 36 & 2 & 2 & 1 & 33 & 2 & 40 & 1 & 3 & 40 & 4 & 3 & 70 & 45.9 & Over Stressed \\
\hline & L3 & 4 & 4 & 3 & 3 & 2 & 64 & 3 & 2 & 2 & 47 & 3 & 60 & 2 & 3 & 50 & 4 & 3 & 70 & 58.6 & Stressed \\
\hline & L4 & 4 & 4 & 3 & 2 & 1 & 56 & 2 & 2 & 2 & 40 & 3 & 60 & 2 & 3 & 50 & 4 & 3 & 70 & 56.4 & Stressed \\
\hline & L5 & 4 & 3 & 2 & 1 & 1 & 44 & 2 & 1 & 1 & 27 & 2 & 40 & 2 & 3 & 50 & 4 & 3 & 70 & 48.6 & Over Stressed \\
\hline \multirow{5}{*}{ 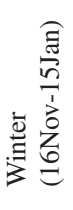 } & L1 & 5 & 4 & 4 & 3 & 2 & 72 & 4 & 3 & 3 & 67 & 3 & 60 & 2 & 3 & 50 & 4 & 3 & 70 & 62.9 & Good \\
\hline & $\mathrm{L} 2$ & 5 & 2 & 2 & 1 & 1 & 44 & 4 & 2 & 1 & 47 & 2 & 40 & 2 & 2 & 40 & 4 & 3 & 70 & 49.1 & Over Stressed \\
\hline & L3 & 5 & 4 & 3 & 3 & 3 & 72 & 4 & 4 & 2 & 67 & 3 & 60 & 2 & 3 & 50 & 4 & 3 & 70 & 62.8 & Good \\
\hline & $\mathrm{L} 4$ & 5 & 3 & 3 & 3 & 2 & 64 & 4 & 3 & 1 & 53 & 3 & 60 & 1 & 3 & 40 & 4 & 3 & 70 & 57.1 & Stressed \\
\hline & L5 & 5 & 3 & 3 & 1 & 1 & 52 & 4 & 2 & 2 & 53 & 2 & 40 & 2 & 3 & 50 & 4 & 3 & 70 & 53.8 & Stressed \\
\hline \multirow{5}{*}{ 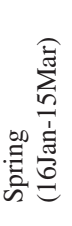 } & L1 & 5 & 4 & 4 & 2 & 3 & 72 & 4 & 4 & 4 & 80 & 3 & 60 & 2 & 3 & 50 & 4 & 3 & 70 & 64.8 & Good \\
\hline & $\mathrm{L} 2$ & 5 & 2 & 2 & 1 & 2 & 48 & 5 & 4 & 2 & 73 & 2 & 40 & 2 & 3 & 50 & 4 & 3 & 70 & 56.2 & Stressed \\
\hline & L3 & 5 & 4 & 3 & 2 & 3 & 68 & 5 & 4 & 3 & 80 & 3 & 60 & 2 & 3 & 50 & 4 & 3 & 70 & 64.2 & Good \\
\hline & $\mathrm{L} 4$ & 5 & 3 & 3 & 2 & 3 & 64 & 5 & 4 & 3 & 80 & 3 & 60 & 2 & 3 & 50 & 4 & 3 & 70 & 63.6 & Good \\
\hline & L5 & 5 & 3 & 3 & 1 & 2 & 56 & 4 & 2 & 2 & 53 & 2 & 40 & 2 & 3 & 50 & 4 & 3 & 70 & 54.4 & Stressed \\
\hline \multirow{5}{*}{ 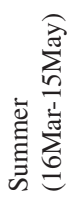 } & L1 & 4 & 3 & 3 & 2 & 2 & 56 & 3 & 2 & 3 & 53 & 2 & 40 & 2 & 3 & 50 & 4 & 3 & 70 & 54.4 & Stressed \\
\hline & L2 & 3 & 0 & 1 & 0 & 0 & 16 & 1 & 1 & 1 & 20 & 0 & 0 & 2 & 2 & 40 & 4 & 3 & 70 & 32.9 & Critical \\
\hline & L3 & 4 & 3 & 2 & 2 & 2 & 52 & 2 & 2 & 2 & 40 & 2 & 40 & 2 & 3 & 50 & 4 & 3 & 70 & 51.8 & Stressed \\
\hline & $\mathrm{L} 4$ & 4 & 2 & 2 & 1 & 1 & 40 & 2 & 1 & 1 & 27 & 2 & 40 & 2 & 2 & 40 & 4 & 3 & 70 & 45.5 & Over Stressed \\
\hline & L5 & 2 & 1 & 1 & 0 & 1 & 20 & 2 & 1 & 1 & 27 & 1 & 20 & 2 & 2 & 40 & 4 & 3 & 70 & 38.5 & Critical \\
\hline
\end{tabular}

only at upstream location (L1) and this location also becomes stressed during the summer season. All other locations are under poor river health category, varying from 'Stressed' to 'Critical' levels. Fig. 3 shows the variation of RHIas graphs and Fig.4 gives the coloured pictorial representation of River Health Condition (RHC) as quality pentagon and scores under various categories for four seasons during 2016-17 and 2017-18 at different locations.

From Fig. 3, based on River Health Index (RHI), it appears that river health is at its lowest levels during the summer season, which improves during post-monsoon months. During winter, the river health improves further and attains its best levels during the spring season (16Jan$15 \mathrm{Mar})$. It is also observed that near Varanasi, the river health at most of the Ghats has improved in 2017-2018 with respect to the year 2016-2017. Better river health during the spring season (16Jan-15Mar) could be due to increasing temperature and other environmental factors. Among OEB parameters, BOD and faecal coliforms in the riverine environment are at lowest levels in the spring in comparison to other seasons. The OEB group score at L1 and L3 is Very Good and Good respectively during the spring season. All the nutrient parameters decreased during the spring season which increases the NT score and all locations except L5 are in 'Very Good' category. As water quality improves for these parameters, there is an increase in the population and diversity of biotic indicators. The population of pollution sensitive species increase, which increases the scores of biotic indices. The algal group scores at L1, L3 and L4 are in 'Stressed' range and L2 \& L5 are in 'Critical' condition during the spring season. It is noted that locations L1, L3 and L4 show presence of the genera Flagilaria, Spirogyra, 

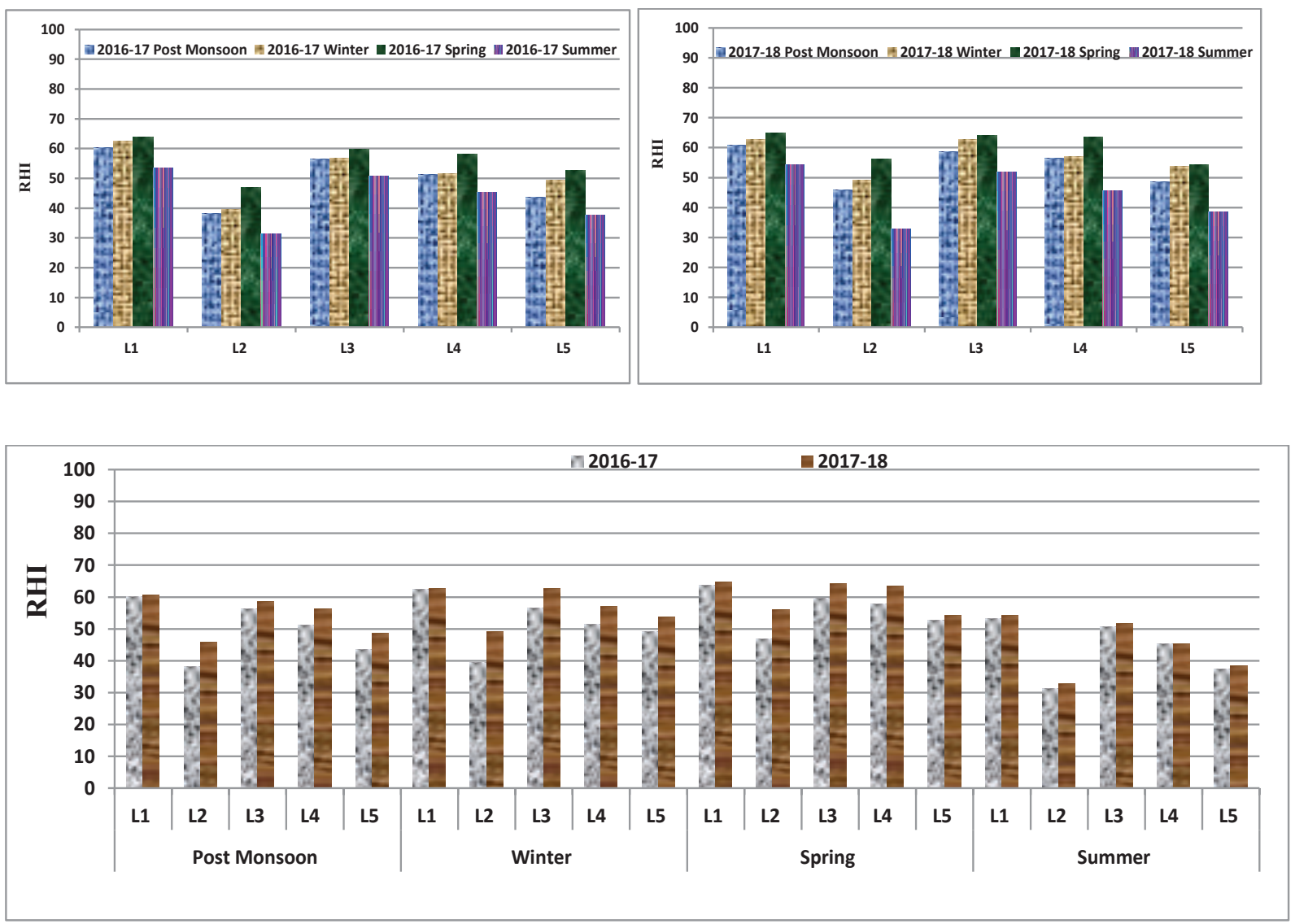

Fig. 3: Variation of River Health Index (RHI) for River Ganga at different locations near Varanasi during 2016-2017 and $2017-2018$.

Staurastrum, etc. of algae. Venkateswarlu \& Reddy (1985) reported that the abundance of green algal flora like Zygnema, Spirogyra, Mougeotia, Euastrum, Staurastrum, etc. indicate less polluted water. Among macroinvertebrates, presence of moderately sensitive species such as Baetidae, Culicidae, Dytiscidae, Elmidae, Hydrophilidae, Psychodidae families at locations L1, L3 and L4 indicate good quality of water at these locations. Adakole (2001) also noted that the presence of Mayflies and Caddisflies reflects clean water. This increase in biotic scores improves the River Health Index (RHI). A higher RHI indicated a better River Health Condition (RHC).

The water quality and river health are found at its lowest levels during summer (16Mar-15May), possibly because with the onset of summer, the temperature starts rising and the DO starts decreasing due to high microbial activities involved in organic matter decomposition. The decrease in DO and an increase in BOD and faecal coliforms reduce the OEB group score. Due to low dilution and increased pollution, nutrient concentration also increases. The decrease in water quality affects the aquatic biota present. Due to low level of DO, reduced discharge and increased concentration of pollutants, sensitive biotic species decreases and there is an increase in the number of pollution resistant species (Genter \& Lehman 2000, Biosson \& Perrodin 2006). This increase in pollution resistant species decreases the biotic indices score which lowers the RHI value, indicating deteriorating river health condition. At L2 (the confluence of Assi Nala with Ganga) and L5 (the confluence of Varuna with Ganga), large amounts of sewage are added to River Ganga. The presence of algal genera such as Ankistrodesmus, Euglena, Navicula, Nitzschia, Oscillatoria, Scenedesmus, etc. at these locations during the period is indicative of polluted water, as noted by Patrick (1965) and Palmer (1969) who concluded that Ankistrodesmus, Euglena, Navicula, Scenedesmus, Stigeoclonium, Oscillatoria, Chlamydomonas and Nitzschia are highly pollution tolerant genera and found in organically polluted waters. Pearsall (1932) was the first to establish a correlation between blue-green algae and organic pollution tolerant species of diatoms such as Anabaena, Chlorella, Closterium, Cosmarium, Eudorina, Melosira, Navicula, Pandorina, Scenedesmus and Spirulina. Rai et al. (2008), Das et al. (2007), Sanap (2007), Jafari \& Gunale (2006), Goel et al. 

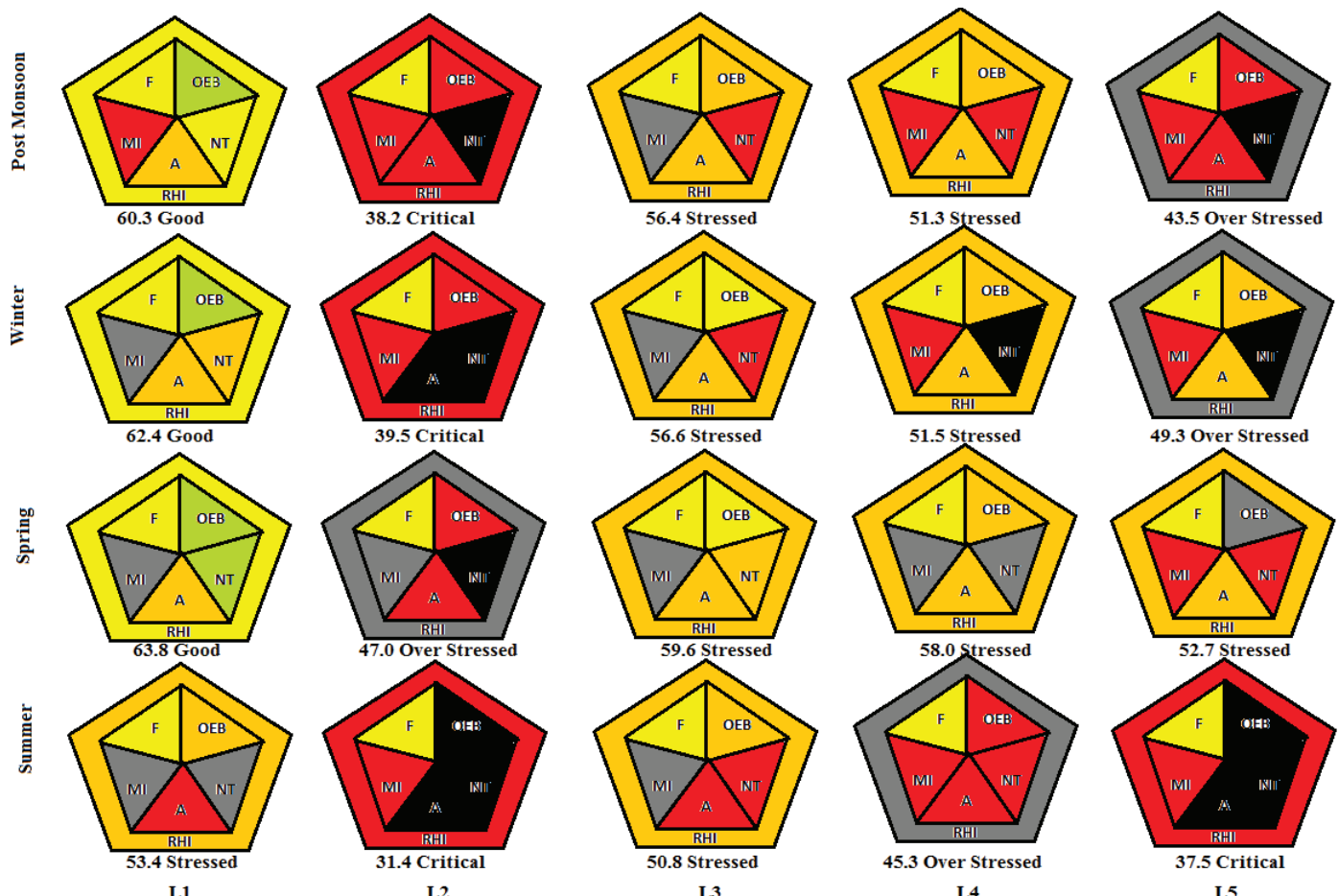

2016-17
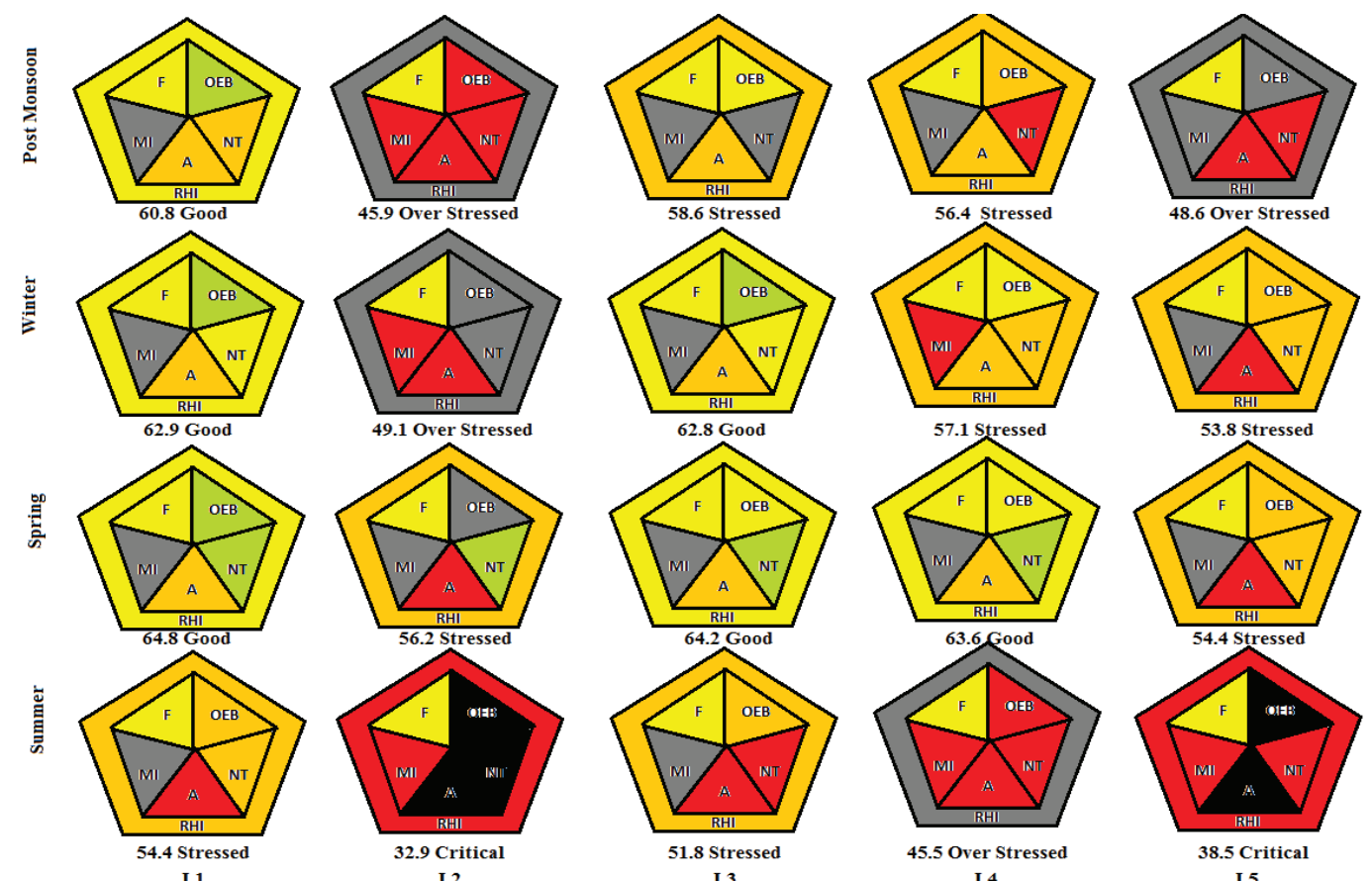

2017-18
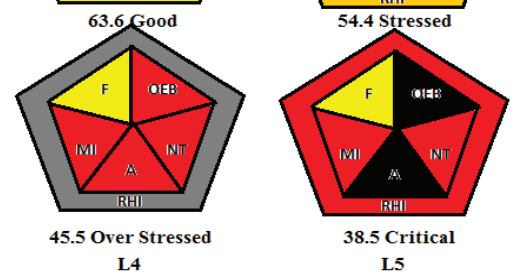

Fig. 4: Pictorial representation for River Ganga at different locations near Varanasi during 2016-2017 and 2017-2018. 
(1986), Gunale \& Balakrishnan (1981)and Ratnasabapathy (1975) also suggested that the presence of these pollution tolerant species indicate polluted waters. At locations, L2, L4 and L5 the repeated presence of macroinvertebrates Oligochaeta (Tubificids, Tubifex) Chironomids (midge larvae), Physidae and Muscidae indicate polluted water which is in accordance with the findings of Adakole (2001) who reported that certain tubificids (especially Tubifex tubifex and Lumnodrilus hoffmeisteri) or midge larva of the genus Chironomus or Eristalis larvae or class Oligochaeta can reflect low DO levels and high organic concentration in an area. The macroinvertebrate species which are pollution tolerant are expected to be more dominant in polluted waters (Sallenave 2015). Sharma et al. (2014) gave the order of disappearance of organisms due to continuous increase in pollution as Plecoptera (stoneflies): Ephemeroptera (mayflies, damselflies etc): Trichoptera (caddisflies): Gammarus (freshwater shrimp): Asellus (water hog louse): Chironomidae (blood worms): Oligochaeta (tubificid worms).

It is evident from Fig 4. that at upstream of Varanasi (L1), the health of River Ganga is in 'Good' condition. As it enters the city, the first point is C/O of Assi Nala (L2) where a large quantity of sewage is being added to the river and the health condition varies at this point between 'Over Stressed' and 'Critical' during various seasons of the year. In OEB group category, DO decreases and there is an increase in the $\mathrm{BOD}, \mathrm{COD}$ and faecal coliforms. In Nutrient group, there is an increase in $\mathrm{NH}_{3}-\mathrm{N}, \mathrm{TN}$ and $\mathrm{TP}$ concentration.

At Dashashwamedh Ghat (L3), both OEB and NT group parameters seem to have improved possibly due to self-purification, or some physico-chemical reactions, and the health comes in the 'Good' category during winter and spring and 'Stressed' condition during summer. However, as it moves downstream to Raaj Ghat (L4) and C/O Varuna with Ganga (L5), the concentration of COD and faecal coliform parameters of OEB group and TN and TP parameters of NT group increase. Consequently, the river health again comes under 'Stressed' to 'Critical' condition at these points.

CPCB (2018) reported the biological water quality of River Ganga at Varanasi in moderate pollution range in May 2017 and March 2018. The results of these analyses are also reflective of similar conclusions, although with a deeper diagnostic approach.

Using the observational data for two years (Sept 2016May 2018), the river health condition of Ganga near Varanasi may be categorized as 'Good' to 'Stressed' at upstream of Varanasi (L1), and near Dashaswamedhghat (L3), 'Stressed' to 'Overstressed' near Rajghat (L4), and 'Overstressed' to 'Critical' near the confluence of river Assi with Ganga (L2) and Varuna with Ganga (L5). For comparison, the river health condition of Ganga as categorized from the framework presented in this study, based on RHI calculation at Rishikesh (near Laxman Jhoola) in winter and spring 2019 is 'Very Good' and 'Excellent' respectively, as shown in Fig. 5.

Thus, the framework for an understanding of river health condition, based on river health index (RHI) calculation, as discussed in the present study appears to give good results capable of describing the riverine condition in a simple but scientifically sound manner. Its coloured representation of scores for five indicators groups of river water quality and overall river health condition makes it simpler to the scientific community for diagnostic and corrective step purposes.

\section{CONCLUSIONS}

In the present study, a framework for a scientific assess-

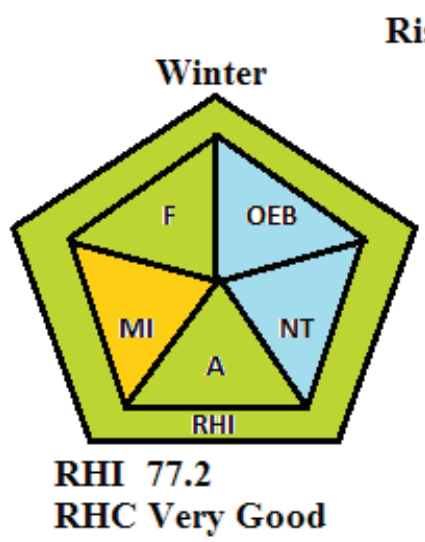

Rishikesh

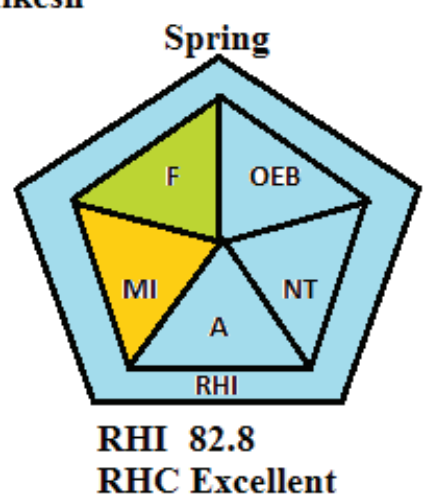

Fig. 5: RHI and RHC of Laxman Jhoola, Rishikesh during winter and spring. 
ment of river health condition involving eight water quality parameters and five biotic indices, have been proposed and tested. Water quality parameters have been divided into two groups: i. Organo-Electrolytic-Bacterial (OEB) and ii. Nutrients (NT). Also, one algal (A), two macroinvertebrates(MI) and two fish (F) indices have been used to calculate River Health Index (RHI) on a 0-100 scale. Based on RHI value, River Health Condition (RHC) has been classified from Sick/Dead to Excellent and represented through a coloured circumscribed pentagon, each side depicting the condition of river health for one category of riverine water quality. This framework has been used to assess the health of River Ganga near Varanasi through measurement of relevant water quality parameters and evaluation of biotic indices for four seasons (post-monsoon, winter, spring and summer) continued for two years. Based on RHI calculation, the health of River Ganga near Varanasi was found to be slightly improving in the year 2017-2018 for 2016-2017. River health is observed unstable during the post-monsoon period and found its best levels during the spring season (16Jan-15March). At polluted locations of the river, pollution resistant and at clean/less polluted locations sensitive or moderately sensitive algae and macroinvertebrate community are present. Pollution resistant species of algae and macroinvertebrate were observed near the confluence of river Assi (L2) and Varuna with Ganga (L5), and clean locations such as SaamneGhat (L1) and DashashwamedhGhat (L3) and moderately polluted location near RaajGhat (L4) showed the presence of sensitive or moderately sensitive species. The confluence of river Assi (L2) and the confluence of Varuna with Ganga (L5) were found to be organically polluted. In spatial terms, the river health is generally acceptable and found as 'Good' near Saamne Ghat, followed by Dashashwamedh Ghat and Raaj Ghat in Varanasi stretch. Overall, it varied between 'Good' to 'Overstressed' conditions during the full year, except near Assi and Varunariver confluence points, where it is found to be 'Critical'. The Indicator Group Score based approach of River Health Index (RHI) calculation gives insights for identification of critical parameters and strategic plan preparation for restoration. The analyses indicate that RHI can be used as a tool to understand river health conditions.

To improve the River Health Condition, the restoration plan should focus on parameters whose group score are below acceptable (less than 3 on the 0-5 scale). Nutrients (NT) group of parameters appear the most critical and algal score seems the worst affected for river health near Assi and Varuna confluence points. Highly polluted waters coming from these rivers need to be checked and controlled. Instream treatment such as ponding and active algal harvesting to reduce organic pollutants and nutrients load, use of boulders to create roughness in the bed, fountains for aeration, and floating algal mats in the streams can be used to improve the water quality of Assi and Varunarivers before they meet River Ganga. With these initiatives, the river health condition of Ganga near Varanasi may be improved substantially and maintain it at acceptable levels all around the year.

\section{REFERENCES}

ACEDP 2011. Australia-China Environment Development Partnership River Health and Environmental Flow in China. Assessment of River Health in the Liao River Basin (Taizi Sub-Catchment) Project Report: Prepared by the International Water Centre and the Chinese Research.

Adakole, J.A. 2001. The effect of domestic agricultural and industrial effluents on the water quality and biota of Bindare Stream, Zaria-Nigeria.Ph.D. Thesis, Deptt. of Biological Sciences, Ahmadu Bello University, Zaria.

Allan, J.D. 1995. Stream Ecology: Structure and Function of Running Waters, 2nd Edition. Kluwer Academic Publishers. The Netherlands.

Anon 2000. Australian and New Zealand Guidelines for Fresh and Marine Water Quality. Volume 1, The Guidelines, Australian and New Zealand Environment and Conservation Council, Agriculture and Resource Management Council of Australia and New Zealand.

APHA 2005. Standard Methods for the Examination of Water and Wastewater. 21st Edition, American Public Health Association/American Water Works Association/Water Environment Federation, Washington DC.

Barbour, M.T., Gerritsen, J., Snyder, B.D. and Stribling, J.B. 1999. Rapid Bioassessment Protocols for Use in Streams and Wadeable Rivers: Periphyton, Benthic Macroinvertebrates andFish, Second Edition. EPA 841-B-99-002. U.S. Environmental Protection Agency, Office of Water, Washington, D.C.

Bhardwaj, R.M. 2005. Water Quality Monitoring in India- Achievements And Constraints. IWG-Env, International Work Session on Water Statistics, Vienna.

Biosson, J. C. and Perrodin, Y. 2006. Effects of road runoff on biomass and metabolic activity of periphyton in experimental streams. Journal of Hazardous Materials, 132(2-3): 148-154.

Leigh, C., Qu, X., Zhang, Y., Kong, W., Meng, W., Hanington, P., Speed, R., Gippel, C., Bond, N., Catford, J., Bunn, S. and Close, P. 2012. Assessment of River Health in the Liao River Basin (Taizi Subcatchment) International Water Centre, Brisbane, Australia.

CPCB 2002. Water Quality Criteria and Goals. Feb 2002, Central Pollution Control Board, Delhi.

CPCB 2015. A Concept and Plan Conservation of Water Quality of Ganga: A Segmental Approach Proposed Primary Water Criteria For Bathing Water. Dec 2015, Central Pollution Control Board, Delhi.

CPCB 2018. Biological Water Quality Assessment of the River Ganga (2017-18). Bio-Science Division, Central Pollution Control Board, Ministry of Environment, Forest \& Climate Change, Govt. of India, Delhi, June 2018.

Das, S.K., Biswas, D. and Roy, S. 2007. Phytoplanktonic community of organically polluted tropical reservoirs in Eastern India. Chinese Journal of Applied Environmental Biology, 13(4): 449-453.

Das, M.K., Sharma, A.P., Vass, K.K., Tyagi, R.K., Suresh, V.R., Naskar, M. and Akolkar, A.B. 2013. Fish diversity, community structure and ecological integrity of the tropical RiverGanges, India, Aquatic Ecosystem Health \& Management, 16(4): 395-407.

De Zwart, D. and Trivedi, R. C. 1994. Manual on Integrated Water Quality Evaluation (Report 802023003). National Institute of Public Health and Environmental Protection (RIVM), Bilthoven, The Netherlands.

Durrand, J. R. and Leveque, C. 1981. Flore et fauna Aqua tyries De 1' AfrqueSaholo-Soudamienne edition de 1' office de la Recherche Scientific et Technique actre-marcollectioninitiatins. Documentations Technique No. 45 Paris. 
Dwivedi, A.C., Mishra, A. S., Mayank, P. and Tiwari, A. 2016. Persistence and structure of the fish assemblage from the Ganga River (Kanpur to Varanasi section), India. Journal of Geography \& Natural Disasters, 6: 159 .

EHMP 2010. Ecosystem Health Monitoring Program 2008-09, Annual Technical Report, South East Queensland Healthy Waterways Partnership, Brisbane.

Genter, R.B. and Lehman, R.M. 2000. Metal toxicity inferred from algal population density, heterotrophic substrate use, and fatty acid profile in a small stream. Environmental Toxicology and Chemistry, 19(4): 869-878.

Gerber, A. and Gabriel, M.J.M. 2002. Aquatic Invertebrates of South African Rivers. Field Guide. Volume 1 and II, Institute for Water Quality Studies, Department of Water Affairs and Forestry.

Goel, P.K., Khatavkar, S.D., Kulkarni, A.Y. and Trivedy, R. K. 1986. Limnological studies of a few freshwater bodies in southwestern Maharasthra with special reference to their chemistry and pollution. Poll. Res., 5(2): 79-84.

GRBMP 2015. Ganga River Basin Management Plan. Main Plan Document. Consortium of seven Indian Institute of Technologies (IITs), India.

Gunale, V. R. and Balakrishnan, M.S. 1981. Biomonitoring of eutrophication in the Pavana, Mula and Mutha rivers flowing through Poona. Indian Journal of Environmental Health, 23: 316-322.

Hawkes, H.A. 1979. Invertebrates as indicators of river water quality. In: Biological Indicators of Water Quality, James, A. and Evison L. (Eds.), pp. 2.1-2.45, Chichester, New York, John Wiley and Sons, UK.

Hellawell, J.M. 1986. Biological Indicators of Freshwater Pollution and Environmental Management. Elsevier, London, p. 546.

Jafari, N.G. and Gunale, V. R. 2006. Hydrobiological study of algae of an urban freshwater river. Journal of Applied Science and Environment Management, 10(2): 153-158.

Kerkhoff, 2010. Measuring biodiversity of ecological communities. Ecology lab, Biology, 229. http://biology.kenyon.edu/courses/biol229/ diversity.pdf

MEP 2008. Report on the State of the Environment in China 2006, Ministry of Environmental Protection, People's Republic of China.

Merritt, R.W. and Cummins, K.W. 1996. An Introduction to the Aquatic Insects of North America, $3^{\text {rd }}$ edition. Dubuque, J. O, Kendall-Hunt, Iowa.

MoWR 2014. Ganga Basin-Version 2.0. Ministry of Water Resources, Govt. of India.

Nandan, S.N. and Patel, R. J. 1986. Assessment of water quality of Vishwamitra river by algal analysis. Indian J. Environ. Health, 29(2): 160-161.

Needham, J. G. and Needham, P. R. 1969. A Guide to the Study of Freshwater Biology. Holden-Day Inc., San Francisco, 108.

Palmer, C.M. 1969. Composite rating of algae tolerating organic pollution. Journal of Phycology. 5: 78-82.
Patrick, R. 1965. Algae as indicator of pollution. In: Biological Problems in Water Pollution. $3^{\text {rd }}$ Seminar, Bot. A. Tuft. Sanitary Engg. Centre, Cincinnati, Ohio.

https://www.researchgate.net/publication/268375252_ALGAE_AS_AN_ INDICATOR_OF_RIVER_WATER_POLLUTION_-_A_REVIEW

Pearsall, W. H. 1932. Phytoplankton in the English lakes II. Ecol., 22: 241-262.

Pennak, W. P. 1978. Freshwater Invertebrates of the United State. Oxford, Ronald.

Pennak, R. W. 1989. Fresh-water Invertebrates of the United States: Protozoa to Mollusca. 3rd. ed. John Wiley and Sons, New York.

Rai, U.N., Dubey, S., Shukla, O.P., Dwivedi, S. and Tripathi, R.D. 2008. Screening and identification of early warning algal species for metal contamination in freshwater bodies polluted from point and non-point sources. Environmental Monitoring Assessment, 144: 469-481.

Ratnasabapathy, M. 1975. Biological aspects of Wardieburn sewage oxidation pond. Malaysian Science, 3(a): 75-87.

Rosenberg, D.M. and Resh, V.H. 1993. Freshwater Biomonitoring and Benthic Macroinvertebrates. Chapman \& Hall, London, p. 488.

Sallenave, R. 2015. Stream biomonitoring using benthic invertebrates. Circular 677. http://aces.nmsu.edu/pubs/_circulars/CR677.pdf, New Mexico State University 4 Cooperative Extension Service. College of Agricultural, Consumer and Environmental Sciences.

Sanap, R.R. 2007. Hydrobiological studies of Godavari River up to Nandur-Madhmeshwar dam, Dist Nashik, Maharashtra. Ph. D. Thesis, University of Pune, Pune, India.

Sharma, Shailendra, Dawar, Bhavna and Barkale, Shitika 2014. Biomonitoring a biological approach to water quality management. Elixir Bio Diver., 66: 20635-20638.

Singh, P. K. and Saxena, S. 2018.Towards developing a river health index. Ecological Indicators, 85: 999-1011.

Sladecek, V. 1979. Continental systems for the assessment of river water quality. In: Biological Indicators of Water Quality. James, A. and Evison L. (Eds.), John Wiley \& Sons, Chichester.

Tittizer, T.T. and Koth, P. 1979. Possibilities and limitations of biological methods of water analysis. In: James A. and Evison L. (Eds.), Biological Indicators of Water Quality, John Wiley and Sons, Chichester.

Tonapi, G. T. 1980. Freshwater Animals of India- An Ecological Approach. Oxford and IBH Publishing Co., New Delhi, 341.

UNECE 1994. ECE Standard Statistical Classification of Surface Freshwater Quality for The Maintenance of Aquatic Life. The United Nations Economic Commission for Europe.

Venkateswarlu, V. and Reddy, P.M., 1985. Algae as biomonitors in river ecology. In: Symposium on Biomonitoring, State of Environment, New Delhi, India, pp. 183-189.

William, D.P. and Feltmate, B. W. 1992. Aquatic Insects. C.A.B. International, Wallingford Oxon, UK. 